\title{
A detour guide to the Endocrine Society Clinical Practice Guideline on case detection, diagnosis and treatment of patients with primary aldosteronism
}

\author{
Wiebke Arlt \\ School of Clinical and Experimental Medicine, Centre for Endocrinology, Diabetes and Metabolism, University of Birmingham, \\ Birmingham B15 2TT, UK \\ (Correspondence should be addressed to W Arlt; Email: w.arlt@bham.ac.uk)
}

The Endocrine Society (USA) commissions clinical practice guidelines that address topics of major interest to the endocrine community and for which international consensus is urgently needed. As a reflection of the important contributions of European endocrinologists to the field, the Endocrine Society Clinical Guidelines Subcommittee has selected more and more European experts to join their American colleagues and help compiling and reviewing the guidelines before they are issued. However, a mid-term goal of transatlantic collaboration would certainly be the joint release of guidelines that appear concurrently in the Journal of Clinical Endocrinology \& Metabolism and the European Journal of Endocrinology. Until this is achieved, we have actually decided to release a series of invited 'European perspective' commentaries on recently published guidelines and in this issue we will make a start with this commentary on the Clinical Practice Guideline on diagnosis and treatment of primary aldosteronism (1).

Why do we need a clinical practice guideline on primary aldosteronism? Usually guidelines are needed when there have been important changes, primarily in the way we perceive the significance of a disease or when research has gained novel insights into pathogenesis and opens up new options for treatment. Primary aldosteronism has made it into the headlines over the last few years mainly due to the notion that it might be more frequent than previously assumed, possibly affecting up to $10 \%$ of all hypertensive patients $(2,3)$, with a recent large European trial reporting a prevalence of $4.8 \%$ (4). Thus, previous diagnosis rates would not reflect the entirety of a very rare disease but perhaps only the "tip of iceberg', with many others also affected, but currently undiagnosed. With this perceived paradigm change came a change in attitude towards screening for primary aldosteronism. While we previously aimed to establish this diagnosis mainly in patients presenting with hypokalaemic hypertension, more and more physicians took to actively screening their hypertensive patients for the presence of primary hyperaldosteronism. However, given that in western countries currently about $10 \%$ of the population suffers from hypertension, these changes in diagnostic strategy represent a significant risk for our health care systems, potentially generating huge costs with insufficiently assessed gain. Thus, a level-headed look at our management strategy for primary hyperaldosteronism, based on systematic review of available evidence was urgently needed, and this is exactly what these guidelines set out to achieve.

Though all of us wish that guidelines should pave a simple and direct way to properly diagnosing and managing a disease, primary hyperaldosteronism will remain a challenge to the experienced endocrinologist despite the current guidelines. The guideline panel achieved a great deal by systematically pulling together and weighing available evidence. The guideline paper maps out a flowchart through the management process of primary hyperaldosteronism, which I have summarised in Fig. 1. However, in the following few paragraphs, I would like to highlight a few unresolved issues, which unfortunately require more of a detour guide and cannot be easily addressed by a straight forward guideline. Each of these issues relates to one of the four key recommendations of the guideline.

'Case detection: We recommend case detection of primary aldosteronism be sought in higher risk groups of hypertensive patients and those with hypokalemia by determining the aldosterone-renin ratio under standard conditions.'

The guidelines panel clearly defined the patients perceived at higher risk of hyperaldosteronism (Fig. 1), and this is important to avoid mass screening for primary hyperaldosteronism in all hypertensive patients. However, how do we screen for it when it is warranted? We should determine 'the aldosteronerenin ratio under standard conditions', an approach that comes with two significant problems.

First, there are no accepted standard conditions for measuring the aldosterone-to-renin ratio (ARR) and appropriately Table 3 in the guidelines outlines 'a suggested approach' to measurement of the ARR (1). However, that approach is complex, with the major problem being that most of the patients undergoing screening suffer from significant hypertension and therefore are mostly on a combination of multiple anti-hypertensive drugs that are likely to impact on 


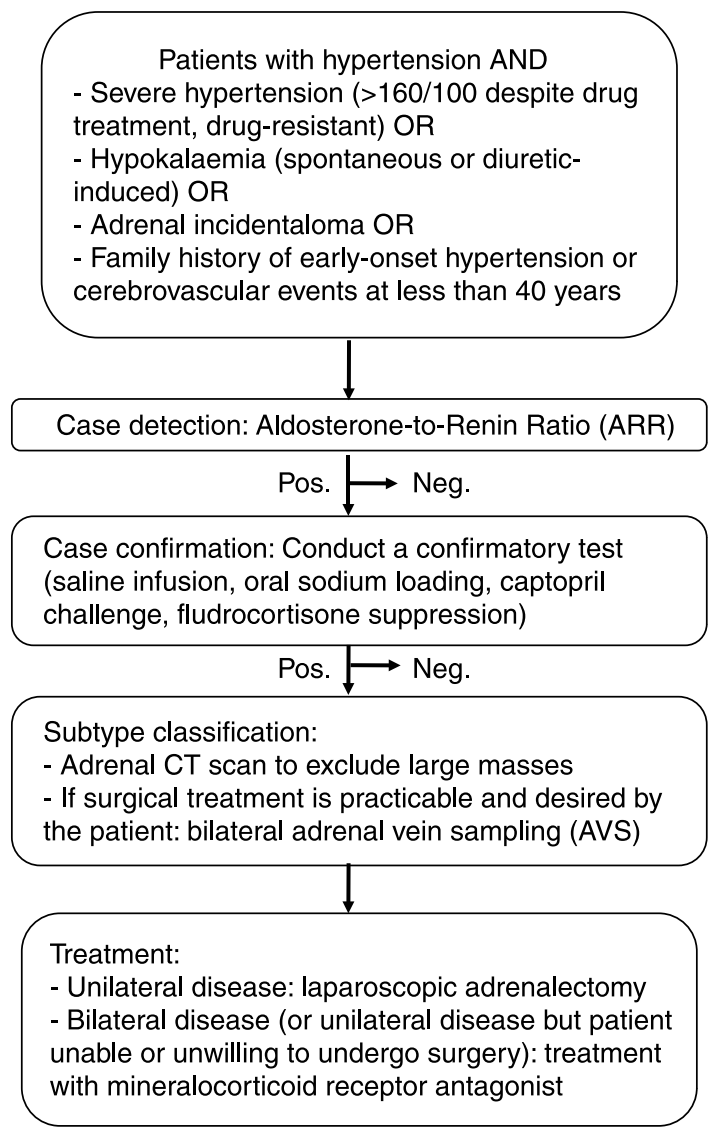

Figure 1 Flowchart outlining the suggested work-up of patients with hypertension and increased risk of hyperaldosteronism according to the Endocrine Society Clinical Practice Guideline on Primary Aldosteronism.

aldosterone, renin or both. The suggested approach is to correct hypokalaemia, liberalise sodium intake and withdraw for at least 4 weeks those drugs that have a major effect on the ARR, such as mineralocorticoid receptor antagonists, amiloride and triamterene. However, if results are not diagnostic, one will need to withdraw more drugs including $\beta$-blockers and angiotensin converting enzyme (ACE) inhibitors for at least 2 weeks to facilitate an informative, repeat ARR test. It is obvious that this comes with significant costs as this approach will require a major monitoring effort in a severely hypertensive patients, equalling multiple outpatient contacts, in some cases even inpatient stays. Most significantly, this approach might put some of our patients with most severe hypertension at risk of hypertensive crisis.

Secondly, once we have measured the ARR and have our numbers to look at, this does not necessarily mean that diagnosis is now straightforward. As acknowledged by the guidelines, there is a "substantial variability in cut-off values' for the ARR and not all researchers agree whether, in addition, there should be a defined cut-off for aldosterone levels. If we want to avoid such a formal cut-off, we will certainly have to accept that the 'likelihood of a false-positive ARR becomes greater when renin levels are very low' (1). A related issue is the assays we actually use to measure aldosterone and specifically renin. Most centres currently measure plasma renin activity, and the measurement of direct renin concentrations, which has the potential to be more accurate and less cumbersome, is still evolving, both with regard to accessibility and methodology. Given the variability in immunometric assays for this target, the move to tandem mass spectrometry for measurement of aldosterone is on the cards and already happening in some places. Europe and specifically the UK is taking an important lead here as centralised assaying of blood samples within the National Health Service allows for widespread, cost-efficient introduction of mass spectrometry. By contrast, in the US, this is still hampered by the fact that the majority of samples are measured in small labs scattered all around the country, which do not have sufficient sample throughput to facilitate the cost-efficient introduction of mass spectrometry, an unfortunate situation several European countries are also faced with.

'Case confirmation: We recommend that the condition be confirmed/excluded by one of four commonly used confirmatory tests.'

Again there are two issues here. I fully accept that even if we are screening for primary hyperaldosteronism in a group at high risk, we need to confirm the diagnosis by a second test. However, I do not think it is necessary to perform a confirmatory test, e.g. in a young patient with severe hypokalaemic hypertension, unequivocal ARR and a $1.5 \mathrm{~cm}$ unilateral adrenal nodule. Such a patient can go straight to surgery as the likelihood that his nodule represents an endocrine inactive adenoma is extremely low. However, we certainly have to take a different view when looking at older patients as the likelihood of an adrenal adenoma is 3\% in 40-year-olds and even $10 \%$ in 70 -year-olds $(5,6)$. Here, we also should have in mind the sensible recommendation of the guideline panel that 'all patients with primary aldosteronism should undergo adrenal computed tomography to exclude adrenocortical carcinoma.' I was saddened by one of my recent cases, a young woman with metastasised adrenocortical carcinoma, who had presented with hypokalaemic hypertension to another centre a couple of years earlier and underwent removal of a 4-cm 'Conn adenoma', followed by discharge with no further follow-up. We should all be alerted by mineralocorticoid hypertension in the context of a larger adrenal mass, and these require a complete endocrine adrenal tumour work-up, as co-secretion of several steroids, as retrospectively confirmed in the above case, is highly indicative of malignancy. 
The second issue many of us have with the recommendation of a confirmatory test is that none of the four tests available, oral sodium loading, saline infusion, fludrocortisone suppression or captopril test, is particularly well validated. Sodium loading and fludrocortisone administration are also not useful in patients with severe, uncontrolled hypertension, and these are many of the patients in question, in particular if we have to take them off their usual medication to facilitate proper biochemical diagnosis. The first step here is to perform well-designed diagnostic trials and directly cross-validate these tests employing large patient cohorts, information we currently lack.

'Subtype classification: We recommend the presence of a unilateral form of primary aldosteronism should be established/excluded by bilateral adrenal venous sampling by an experienced radiologist and, where present, optimally treated by laparoscopic adrenalectomy.'

This is a major problem. For one, this test requires 'an experienced radiologist' which we all agree on. In particular, the anatomic difficulties in catheterising the right adrenal vein results in a high rate of unsuccessful catheterisations and success rates even in highly specialised centres (and thus assumed bias towards reporting of better results) can be as low as 40-70\% (7). In a poignant commentary, my colleagues Paul Stewart \& Bruno Allolio have recently summarised the pitfalls of the assumed 'gold standard' technique of bilateral adrenal venous sampling (7). They quite rightly state that adrenal vein sampling (AVS) fulfils none of the criteria we would expect from a gold standard technique including ease of use (and high success rate), safety (significant complications in 1-2\% even in experienced hands), reproducibility and standardisation (no broad agreement on sampling protocols, diagnostic cut-offs and the role of ACTH stimulation), and sensitivity and specificity (in a recent large series (8) reported as 80 and $75 \%$ respectively and not $100 \%$, as to be expected of a gold standard). Most importantly, Stewart \& Allolio highlight the lack of data on the impact of AVS on clinical outcome, with no prospective, randomised trials available demonstrating improved outcome, and two large retrospective studies reporting no benefit $(9,10)$. Finally, they calculate the costs of implementing AVS as per the recommendations of the guidelines (1): $5 \%$ of hypertensive patients, which represent $10 \%$ of 830 million Europeans, undergoing AVS will result in a European AVS budget of 5.8 billion Euros (7), something our health systems will struggle with. From this we have to conclude that we should make careful use of AVS in patients who are suitable and willing to undergo surgery and after stratifying by age. As outlined above, the incidence of adrenal incidentalomas, in the majority of endocrine inactive adenomas, increases with age. Therefore, I do not think that we should subject all our primary hyperaldosteronism patients under 40 years of age routinely to AVS when there is evidence of a unilateral nodule.

'Treatment: We recommend that patients with bilateral adrenal hyperplasia, or those unsuitable for surgery, optimally be treated medically by mineralocorticoid receptor antagonists.'

Again, we will need more effort here to homogenously establish treatment options across Europe, where eplerenone is not approved for use in hypertension, with limited access in several European countries and thus widespread lack of experience with the use of this newer mineralocorticoid antagonist that might come with the advantage of fewer side effects. Unfortunately, spironolactone, the most commonly used drug, is not a selective mineralocorticoid receptor but also counteracts androgen and progesterone action, therefore potentially resulting in gynaecomastia (11), erectile dysfunction and menstrual irregularities. However, despite the longstanding use of spironolactone, at present there is a dearth of data reported on the true incidence of relevant side effects in larger cohorts and larger scale studies on this important issue are needed. Another hope for the future is aldosterone synthase inhibitors that currently undergo clinical development (12).

Taken together, this means that there are lots of options for Europeans and Americans to join forces, both for compiling much needed guidelines and, most importantly, for setting up joint large-scale studies to address important questions that cannot be answered at present. Our guidelines are only as good as the studies we can base them on. Wearing both my hats as an editor of the European Journal of Endocrinology and as Executive Committee Member of the European Society of Endocrinology, I am looking forward to even closer European-American collaboration!

\section{Declaration of interest}

There is no conflict of interest that could be perceived as prejudicing the impartiality of the research reported.

\section{Funding}

This work was supported by the Medical Research Council (Strategic Grant G0801473, Programme Grant G0900567).

\section{References}

1 Funder JW, Carey RM, Fardella C, Gomez-Sanchez CE, Mantero F, Stowasser M, Young WF Jr \& Montori VM. Case detection, diagnosis, and treatment of patients with primary aldosteronism: an Endocrine Society Clinical Practice Guideline. Journal of Clinical Endocrinology E Metabolism 200893 3266-3281. 
2 Gordon RD, Stowasser M, Tunny TJ, Klemm SA \& Rutherford JC. High incidence of primary aldosteronism in 199 patients referred with hypertension. Clinical and Experimental Pharmacology $\mathcal{E}$ Physiology 199421 315-318.

3 Fardella CE, Mosso L, Gómez-Sánchez C, Cortés P, Soto J, Gómez L, Pinto M, Huete A, Oestreicher E, Foradori A \& Montero J. Primary hyperaldosteronism in essential hypertensives: prevalence, biochemical profile, and molecular biology. Journal of Clinical Endocrinology and Metabolism 200085 1863-1867.

4 Rossi GP, Bernini G, Caliumi C, Desideri G, Fabris B, Ferri C, Ganzaroli C, Giacchetti G, Letizia C, Maccario M, Mallamaci F, Mannelli M, Mattarello MJ, Moretti A, Palumbo G, Parenti G, Porteri E, Semplicini A, Rizzoni D, Rossi E, Boscaro M, Pessina AC, Mantero F \& PAPY Study Investigators. A prospective study of the prevalence of primary aldosteronism in 1,125 hypertensive patients. Journal of the American College of Cardiology 200648 2293-2300.

5 Grumbach MM, Biller BM, Braunstein GD, Campbell KK, Carney JA, Godley PA, Harris EL, Lee JK, Oertel YC, Posner MC, Schlechte JA \& Wieand HS. Management of the clinically inapparent adrenal mass ("incidentaloma"). Annals of Internal Medicine 2003138 424-429.

6 Mansmann G, Lau J, Balk E, Rothberg M, Miyachi Y \& Bornstein SR The clinically inapparent adrenal mass: update in diagnosis and management. Endocrine Reviews 200425 309-340.

7 Stewart PM \& Allolio B. Adrenal vein sampling for primary aldosteronism: time for a reality check. Clinical Endocrinology. 2009. In Press DOI: 10.1111/j.1365-2265.2009.03714.x.
8 Rossi GP, Pitter G, Bernante P, Motta R, Feltrin G \& Miotto D. Adrenal vein sampling for primary aldosteronism: the assessment of selectivity and lateralization of aldosterone excess baseline and after adrenocorticotropic hormone (ACTH) stimulation. Journal of Hypertension 200826 989-997.

9 Seccia TM, Miotto D, De Toni R, Pitter G, Mantero F, Pessina AC \& Rossi GP. Adrenocorticotropic hormone stimulation during adrenal vein sampling for identifying surgically curable subtypes of primary aldosteronism: comparison of 3 different protocols. Hypertension 200953 761-766.

10 Zarnegar R, Lee J, Brunaud L, Lindsay S, Kebebew E, Clark OH \& Duh QY. Good blood pressure control on antihypertensives, not only response to spironolactone, predicts improved outcome after adrenalectomy for aldosteronoma. Surgery 2007 142 921-929.

11 Jeunemaitre X, Chatellier G, Kreft-Jais C, Charru A, DeVries C Plouin PF, Corvol P \& Menard J. Efficacy and tolerance of spironolactone in essential hypertension. American Journal of Cardiology 198760 820-825.

12 Stowasser M. Update in primary aldosteronism. Journal of Clinical Endocrinology and Metabolism 200994 3623-3630.

Received 28 September 2009

Accepted 30 September 2009 